\title{
Free Groups and Subgroups of Finite Index in the Unit Group of an Integral Group Ring*
}

\author{
A. Dooms ${ }^{\dagger} \quad$ E. Jespers $\quad$ M. Ruiz ${ }^{\ddagger}$
}

Ann Dooms \& Eric Jespers

Department of Mathematics

Vrije Universiteit Brussel

Pleinlaan 2

1050 Brussel, Belgium

Email: Ann.Dooms@vub.ac.be efjesper@vub.ac.be
Manuel Ruiz

Dep. Métodos Cuantitativos

e Informáticos

Universidad Politécnica de Cartagena

Paseo Alfonso XIII, 50

30.203 Cartagena, Spain

Email: Manuel.Ruiz@upct.es

\begin{abstract}
In this article we construct free groups and subgroups of finite index in the unit group of the integral group ring of a finite non-abelian group $G$ for which every non-linear irreducible complex representation is of degree 2 and with commutator subgroup $G^{\prime}$ a central elementary abelian 2-group.
\end{abstract}

\section{Introduction}

It is well-known from a result of Borel and Harish Chandra that the unit group of the integral group ring $\mathbb{Z}[G]$ of a finite group $G$ is finitely presented [2]. In case $G$ is abelian, Higman showed that $\mathcal{U}(\mathbb{Z}[G])= \pm G \times F$, a direct product of the trivial units $\pm G$ with a finitely generated free abelian group $F$. However, when $G$ is non-abelian, there is no general structure theorem.

Hartley and Pickel [5] showed that if the unit group of the integral group ring of a finite non-abelian group is not trivial, then it contains a non-abelian

\footnotetext{
${ }^{*}$ Research partially supported by the Onderzoeksraad of Vrije Universiteit Brussel, Fonds voor Wetenschappelijk Onderzoek (Belgium) and Bilateral Scientific and Technological Cooperation BWS 05/07 (Flanders-POland).

${ }^{\dagger}$ Postdoctoraal Onderzoeker van het Fonds voor Wetenschappelijk OnderzoekVlaanderen.

${ }^{\ddagger}$ Research partially supported by the Fundación Séneca of Murcia and D.G.I. of Spain.
} 
free subgroup of rank two. Marciniak and Sehgal constructed in [11] such a subgroup using a non-trivial bicyclic unit $u=1+(1-x) y \widehat{x}$ of $\mathbb{Z}[G]$, where $x, y \in G$ and $\widehat{x}=\sum_{1 \leq i \leq o(x)} x^{i}$, with $o(x)$ the order of $x$. They showed that $\left\langle u, u^{*}\right\rangle$ is a non-abelian free subgroup of $\mathcal{U}(\mathbb{Z}[G])$, where $*$ denotes the classical involution on the rational group algebra $\mathbb{Q}[G]$.

It is thus a natural question to ask whether $\langle u, \varphi(u)\rangle$ is free in case $\varphi$ is an arbitrary involution on $G$. We will solve this question for the class $\mathcal{G}$ consisting of the finite non-abelian groups $G$ for which every non-linear irreducible complex representation is of degree 2 and with commutator subgroup $G^{\prime}$ a central elementary abelian 2-group. Due to a result of Amitsur [1] the former condition is equivalent to either $G$ containing an abelian subgroup of index 2 or $G / \mathcal{Z}(G)$ being an elementary abelian 2-group of order 8.

If $e$ is a primitive central idempotent of $\mathbb{Q}[G]$ with $G \in \mathcal{G}$ such that $\mathbb{Q}[G] e$ is non-commutative, then $H=G e \in \mathcal{G}$ and clearly $H^{\prime} \cong C_{2}$. We denote by $C_{n}$ the cyclic group of order $n$. By [10, Lemma 1.4] we know that for an arbitrary finite group $G$ and $p$ a prime, $G / \mathcal{Z}(G) \cong C_{p} \times C_{p}$ is equivalent to $\left|G^{\prime}\right|=p$ and every non-linear irreducible complex representation of $G$ has degree $p$. Thus $H / \mathcal{Z}(H) \cong C_{2} \times C_{2}$. Hence we first will concentrate on groups satisfying the latter property. Furthermore, we also will characterize when two arbitrary bicyclic units generate a free group.

Besides constructing free groups in the unit group of an integral group ring, finding generators for a subgroup of finite index is an important step in understanding the structure of the unit group. When the non-commutative simple components of $\mathbb{Q}[G]$ are of a so-called exceptional type, they are an obstruction to construct in a generic way generators of a subgroup of finite index in the unit group $\mathcal{U}(\mathbb{Z}[G]$ ) (Problem 23 in [15]). For details we refer the reader to [7], [13] and [15].

In [3] $\varphi$-unitary units were introduced to overcome this difficulty for finite groups $G$ of type $G / \mathcal{Z}(G) \cong C_{2} \times C_{2}$ (and also for all groups up to order 16). These $\varphi$-unitary units together with the Bass cyclic units generate a subgroup of finite index in the unit group of $\mathbb{Z}[G]$ and we will extend this result to groups in the class $\mathcal{G}$. Recall that for $g \in G$ with $o(g)=n$ and $1<k<n, \operatorname{gcd}(k, n)=1$, a Bass cyclic unit of $\mathbb{Z}[G]$ is of the form $b(g, k)=\left(\sum_{j=0}^{k-1} g^{j}\right)^{\phi(n)}+\frac{1-k^{\phi(n)}}{n} \hat{g}$, where $\phi$ is the Euler's function.

It is worth mentioning that from the classification in [12, Theorem 3.3] it follows that the class $\mathcal{G}$ contains for example the finite groups of Kleinian type with central commutators. For the finite groups $G$ of Kleinian type there exist geometrical methods [12] that allow to compute a presentation by 
generators and relations for a subgroup of finite index in $\mathcal{U}(\mathbb{Z}[G])$. Although, it is very hard to accomplish these calculations, several examples have been calculated in [12]. Hence we need to obtain more algebraic information on the structure of the unit group $\mathcal{U}(\mathbb{Z}[G])$ of such groups $G$ of Kleinian type.

\section{Free Subgroups}

To investigate the group $\langle u, \varphi(u)\rangle$ where $u$ is a non-trivial bicyclic unit and $\varphi$ is an arbitrary involution on a finite group $G$ we will make use of the following criterion.

Theorem 2.1. [14, 9, Proposition 2.4] Let $A$ be a $\mathbb{Q}$-algebra which is a direct product of division rings and $2 \times 2$-matrix rings over subfields $k$ of $\mathbb{C}$.

Let $a, b \in A$ be such that $a^{2}=b^{2}=0$, then

1. if $a b$ is nilpotent, then $\langle 1+a, 1+b\rangle$ is torsion-free abelian,

2. if $a b$ is not nilpotent and if for some projection $\rho$ of $A$ onto a simple component $M_{2}(k)$ we have that $|\operatorname{Tr}(\rho(a b))| \geq 4$, then $\langle 1+a, 1+b\rangle$ is free of rank 2, where $\operatorname{Tr}$ denotes the ordinary trace function on matrices.

\subsection{Preliminaries}

Let $G$ be a finite group that is not Hamiltonian and such that

$$
G / \mathcal{Z}(G) \cong C_{2} \times C_{2}
$$

Note that by [4, Proposition III.3.6] the latter is equivalent to $G$ having a unique non-identity commutator $s$ and for $x, y \in G$ one has that $x y=y x$ if and only if $x \in \mathcal{Z}(G)$ or $y \in \mathcal{Z}(G)$ or $x y \in \mathcal{Z}(G)$. The last property is the so called lack of commutativity property. Note that $s$ is central of order 2 .

Take $x, y \in G$ with $s=(x, y) \notin\langle x\rangle$, then $u=1+(1-x) y \widehat{x}$ is a non-trivial bicyclic unit of $\mathbb{Z}[G]$. Clearly $x^{2}, y^{2} \in \mathcal{Z}(G)$ and we can write $G=\langle x, y, \mathcal{Z}(G)\rangle$. It is readily verified that an involution $\varphi$ on $G$ has to be of one of the following types:

$$
\varphi_{1}:\left\{\begin{array}{l}
x \mapsto z_{1} x \\
y \mapsto z_{2} y
\end{array} \varphi_{2}:\left\{\begin{array}{l}
x \mapsto z_{1} x \\
y \mapsto z_{2} x y
\end{array} \varphi_{3}:\left\{\begin{array}{l}
x \mapsto z_{1} y \\
y \mapsto z_{2} x
\end{array} \varphi_{4}:\left\{\begin{array}{l}
x \mapsto z_{1} x y \\
y \mapsto z_{2} y
\end{array}\right.\right.\right.\right.
$$

for some $z_{1}, z_{2} \in \mathcal{Z}(G)$. The natural extension of $\varphi$ to a $\mathbb{Q}$-linear involution on $\mathbb{Q}[G]$ is also denoted by $\varphi$. Consider the images of the bicyclic unit $u$ 
under the mentioned involutions $\varphi$. Since $\widehat{g}=\widehat{g^{2}}(1+g)$ for a non-central $g \in G$, we obtain that

$$
u=1+\widehat{x^{2}}(1-x) y(1-s) \text { and } \varphi(u)=1+\widehat{\varphi(x)^{2}}(1+\varphi(x)) \varphi(y)(1-s) .
$$

Investigating the structure of $\langle u, \varphi(u)\rangle$ forces us to look at the non- commutative simple components of $\mathbb{Q}[G]$, thus the simple components of $\mathbb{Q}[G]\left(\frac{1-s}{2}\right)$. By $\left[4\right.$, Proposition VII.2.1] the primitive central idempotents of $\mathbb{Q}[G]\left(\frac{1-s}{2}\right)$ are precisely the elements of the form $e=\widetilde{H}\left(\frac{1-s}{2}\right)$, where $H$ is a subgroup of $\mathcal{Z}(G)$ not containing $s$ and such that $\mathcal{Z}(G)=\langle H, c\rangle$ for some $1 \neq c \in \mathcal{Z}(G)$. Furthermore, if $\mathcal{Z}(G) / H$ has order $m$, with $m>1$ then $\mathcal{Z}(\mathbb{Q}[G]) e \cong \mathbb{Q}\left(\xi_{m}\right)$.

Recall that for a subgroup $H$ of a finite group we denote by $\widetilde{H}$ the idempotent $\frac{1}{|H|} \sum_{h \in H} h$ of $\mathbb{Q}[G]$. Recall that $\widetilde{H}$ is central precisely when $H$ is normal in $G$.

Theorem 2.2. Let $\varphi$ be an involution on a finite group $G$ that is not Hamiltonian and such that $G / \mathcal{Z}(G) \cong C_{2} \times C_{2}$. Let $x, y \in G$ be such that $u=1+(1-x) y \widehat{x}$ is a non-trivial bicyclic unit (thus $s=(x, y) \notin\langle x\rangle)$.

Put $T=\left\langle x^{2}, \varphi(x)^{2}, \varphi(x) x^{-1}\right\rangle=\left\langle x^{2}, \varphi(x) x^{-1}\right\rangle$ in case $\varphi(x) x^{-1}$ is central, otherwise put $T=\left\langle x^{2}, \varphi(x)^{2}\right\rangle$.

Then $\langle u, \varphi(u)\rangle$ is a free group of rank two if and only if $s \notin T$. Otherwise, it is a torsion-free abelian group.

Proof. Let $e$ be an arbitrary primitive central idempotent of $\mathbb{Q}[G]\left(\frac{1-s}{2}\right)$. Then $e=\widetilde{H}\left(\frac{1-s}{2}\right)$ for some subgroup $H$ of $G$ as mentioned above. Put $a=\widehat{x^{2}}(1-x) y(1-s)$ and $b=\widehat{\varphi(x)^{2}}(1+\varphi(x)) \varphi(y)(1-s)$. Then

$$
a b\left(\frac{1-s}{2}\right)=\widehat{4 x^{2}} \widehat{\varphi(x)^{2}}(1+s x) y(1+\varphi(x)) \varphi(y)\left(\frac{1-s}{2}\right) .
$$

If $\varphi=\varphi_{1}$ or $\varphi_{2}$, then

$$
a b\left(\frac{1-s}{2}\right)=\widehat{4 x^{2}} \widehat{z_{1}^{2} x^{2}} z_{2} y^{2}\left(1+z_{1}\right)(1+s x)\left(\frac{1-s}{2}\right) .
$$

If $\varphi=\varphi_{3}$, then

$$
a b\left(\frac{1-s}{2}\right)=\widehat{4 x^{2}} \widehat{z_{1}^{2} y^{2}} z_{2} z_{1}^{-1}(1+s x)\left(1+z_{1} y\right)\left(z_{1} y\right) x\left(\frac{1-s}{2}\right)
$$

If $\varphi=\varphi_{4}$, then

$$
a b\left(\frac{1-s}{2}\right)=\widehat{4 x^{2}} \widehat{\left(z_{1} x y\right)^{2}} z_{2} y^{2}(1+s x)\left(1+z_{1} y\right)\left(\frac{1-s}{2}\right) .
$$


Put

$$
d_{1}=d_{2}=\widehat{4 x^{2}} \widehat{z_{1}^{2} x^{2}} z_{2} y^{2}\left(1+z_{1}\right)
$$

and

$$
d_{3}=\widehat{4 x^{2}} \widehat{z_{1}^{2} y^{2}} z_{2} z_{1}^{-1}, \quad d_{4}=\widehat{4 x^{2}} \widehat{\left(z_{1} x y\right)^{2}} z_{2} y^{2} .
$$

Now $\mathcal{Z}(\mathbb{Q}[G]) \widetilde{H}\left(\frac{1-s}{2}\right) \cong \mathbb{Q}\left(\xi_{m}\right)$ and thus the central torsion units $x^{2} e, y^{2} e$, $z_{1} e, z_{2} e,(x y)^{2} e$ belong to $\left\langle\xi_{m}\right\rangle$, where $m$ is the order of $\mathcal{Z}(G) / H$. It follows in particular that $d_{i} e \in \mathbb{Q}\left(\xi_{m}\right)$ for $1 \leq i \leq 4$. Furthermore, $d_{1} e \neq 0$ if and only if $\widehat{x^{2}} e \neq 0, \widehat{z_{1}^{2} x^{2}} e \neq 0$ and $z_{1} e \neq-e$, while $d_{3} e$ and $d_{4} e$ are non-zero if and only if $\widehat{x^{2}} e \neq 0, \widehat{\varphi(x)^{2}} e \neq 0$.

Write $x^{2} e=\xi_{m}^{i}$ for some $i \geq 0$. Hence

$$
\widehat{x^{2}} e=k \widehat{\xi_{m}^{i}},
$$

where $k=o\left(x^{2}\right) / o\left(\xi_{m}^{i}\right)$ and $\widehat{\xi_{m}^{i}}=\sum_{j=0}^{o\left(\xi_{m}^{i}\right)-1} \xi_{m}^{i j}$. Now $\widehat{\xi_{m}^{i}} \neq 0$ if and only if $\xi_{m}^{i}=1$. Hence $\widehat{x^{2}} e \neq 0$ if and only if $x^{2} e=e$. If this is the case, then

$$
\widehat{x^{2}} e=o\left(x^{2}\right)=\frac{o(x)}{2} .
$$

Similarly, we deduce that $\widehat{\varphi(x)^{2}} e \neq 0$ if and only if $\varphi(x)^{2} e=e$. If this is the case, then

$$
\widehat{\varphi(x)^{2}} e=o\left(x^{2}\right)=\frac{o(x)}{2} \text {. }
$$

Hence

$$
d_{1} e \neq 0 \text { if and only if } x^{2} e=e, z_{1}^{2} x^{2} e=e \text { and } z_{1} e \neq-e,
$$

which is equivalent to $x^{2} e=e$ and $z_{1} e=\varphi(x) x^{-1} e=e$. Thus for $i=1,2,3,4$

$$
d_{i} e \neq 0 \text { if and only if } T \subseteq H,
$$

where $T$ is as in the statement of the Theorem.

If $s \notin T$, then there exists a primitive central idempotent $e=\widetilde{H}\left(\frac{1-s}{2}\right)$ of $\mathbb{Q}[G]$ so that $H$ contains $T$. In particular all $d_{i} e \neq 0$ and thus $\mathbb{Q}[G] e$ is not a division ring. Since it is four dimensional over its center and simple, the algebra $\mathbb{Q}[G] e$ is a two-by-two matrix ring over its center. It is readily verified, using $x^{2} e=e$, that $\mathbb{Q}[G] e$ has the following set of matrix units

$$
\begin{array}{ll}
E_{11}=\frac{1+x}{2} e & E_{12}=y^{-2} \frac{1+x}{2} y \frac{1-x}{2} e \\
E_{21}=\frac{1-x}{2} y \frac{1+x}{2} e & E_{22}=\frac{1-x}{2} e
\end{array}
$$


With respect to these matrix units one verifies that

$$
x e=\left(\begin{array}{cc}
1 & 0 \\
0 & -1
\end{array}\right) \quad y e=\left(\begin{array}{cc}
0 & \xi_{m}^{j} \\
1 & 0
\end{array}\right) \quad s e=\left(\begin{array}{cc}
-1 & 0 \\
0 & -1
\end{array}\right),
$$

for some $j \geq 0$.

Since $z e \in\left\langle\xi_{m}\right\rangle$ for any $z \in \mathcal{Z}(G)$ we also have that $|z e|=1$. It follows that

$$
\begin{aligned}
& \left|\operatorname{Tr}\left(d_{1}(1+s x) e\right)\right|=\left|4 \frac{o(x)^{2}}{4} 2 \operatorname{Tr}\left(\begin{array}{ll}
0 & 0 \\
0 & 2
\end{array}\right)\right|=4 o(x)^{2} \\
& \left|\operatorname{Tr}\left(d_{3}(1+s x)\left(1+z_{1} y\right)\left(z_{1} y\right) x e\right)\right| \\
& =\left|4 \frac{o(x)^{2}}{4} \operatorname{Tr}\left(\left(\begin{array}{cc}
0 & 0 \\
0 & 2
\end{array}\right)\left(\begin{array}{cc}
1 & \xi_{m}^{k+j} \\
\xi_{m}^{k} & 1
\end{array}\right)\left(\begin{array}{cc}
0 & \xi_{m}^{k+j} \\
\xi_{m}^{k} & 0
\end{array}\right)\left(\begin{array}{cc}
1 & 0 \\
0 & -1
\end{array}\right)\right)\right| \\
& =\left|o(x)^{2} \operatorname{Tr}\left(\begin{array}{cc}
0 & 0 \\
2 \xi_{m}^{k} & -2 \xi_{m}^{2 k+j}
\end{array}\right)\right| \\
& =2 o(x)^{2}
\end{aligned}
$$

and

$$
\begin{aligned}
\left|\operatorname{Tr}\left(d_{4}(1+s x)\left(1+z_{1} y\right) e\right)\right| & =\left|4 \frac{o(x)^{2}}{4} \operatorname{Tr}\left(\left(\begin{array}{ll}
0 & 0 \\
0 & 2
\end{array}\right)\left(\begin{array}{cc}
1 & \xi_{m}^{k+j} \\
\xi_{m}^{k} & 1
\end{array}\right)\right)\right| \\
& =\left|o(x)^{2} \operatorname{Tr}\left(\begin{array}{cc}
0 & 0 \\
2 \xi_{m}^{k} & 2
\end{array}\right)\right| \\
& =2 o(x)^{2} .
\end{aligned}
$$

As $o(x) \geq 2$, Theorem 2.1 gives us that $\langle u, \varphi(u)\rangle$ is free.

If $s \in T$, then for every primitive central idempotent $e=\widetilde{H}\left(\frac{1-s}{2}\right)$ of $\mathbb{Q}[G]\left(\frac{1-s}{2}\right)$ (so $H$ is a subgroup of $\mathcal{Z}(G)$ with $s \notin H$ and $\mathcal{Z}(G) / H$ is cyclic) the group $H$ cannot contain $T$. Hence, by $(2), d_{i} e=0$ for $i=1,2,3,4$ and thus $a b\left(\frac{1-s}{2}\right)=0$, so $a b=0$. Therefore, by Theorem $2.1,\langle u, \varphi(u)\rangle$ is torsion-free abelian.

\section{Remark.}

We note that in the proof of the Theorem it is not essential that $\varphi$ is an involution. The result actually characterizes when the non-trivial bicyclic unit $u_{x, y}=1+(1-x) y \widehat{x}$ and $u_{x^{\prime}, y^{\prime}}^{\prime}=1+\widehat{x^{\prime}} y^{\prime}\left(1-x^{\prime}\right)$ generate a free group, where $x^{\prime}, y^{\prime} \in G$ are such that $G / \mathcal{Z}(G)=\left\langle x^{\prime} \mathcal{Z}(G), y^{\prime} \mathcal{Z}(G)\right\rangle$.

Note that there are six cases to be dealt with; when $x^{\prime}=\varphi(x)$ and $y^{\prime}=\varphi(y)$ with $\varphi$ an involution on $G$ then the cases reduce to the four listed 
in (1). Hence to characterize when $\left\langle u_{x, y}, u_{x^{\prime}, y^{\prime}}^{\prime}\right\rangle$ is free we also have to deal with $x^{\prime}=z_{1} x y, y^{\prime}=z_{2} x$ and $x^{\prime}=z_{1} y, y^{\prime}=z_{2} x y$. These are handled in a similar manner.

Since $u_{x^{\prime}, y^{\prime}}=u_{s x^{\prime}, y^{\prime}}^{\prime}$ we then know when any two bicyclic (of both types) generate a free group.

Theorem 2.3. Let $G$ be a finite group that is not Hamiltonian and such that $G / \mathcal{Z}(G) \cong C_{2} \times C_{2}$ and let $u_{x, y}$ and $u_{x^{\prime}, y^{\prime}}$ be non-trivial bicyclic units.

Denote by $s=(x, y)=\left(x^{\prime}, y^{\prime}\right)$. Put $T=\left\langle x^{2}\right.$, sx $\left.x^{\prime} x^{-1}\right\rangle$ in case $x^{\prime} x^{-1}$ is central, otherwise put $T=\left\langle x^{2}, x^{\prime 2}\right\rangle$.

Then $\left\langle u_{x, y}, u_{x^{\prime}, y^{\prime}}\right\rangle$ is a free group of rank two if and only if $s \notin T$. Otherwise, it is a torsion-free abelian group.

\subsection{The class $\mathcal{G}$}

Recall that the class $\mathcal{G}$ consists of the finite groups $G$ for which every nonlinear irreducible complex representation is of degree 2 and with commutator subgroup $G^{\prime}$ a central elementary abelian 2-group.

Let $G \in \mathcal{G}$ and let $x, y \in G$ be such that $\langle x\rangle$ is not normal in $\langle x, y\rangle$ and thus $u=1+(1-x) y \widehat{x}$ is a non-trivial bicyclic unit. Let $S$ be a hyperplane of the elementary abelian 2-group $G^{\prime}$ not containing $t=(x, y)$. Obviously $\left|(G / S)^{\prime}\right|=2$ and thus by [10, Lemma 1.4] $(G / S) / \mathcal{Z}(G / S) \cong C_{2} \times C_{2}$ and thus the primitive central idempotents of $\mathbb{Q}[G / S]\left(\frac{1-t}{2}\right)$ are given by

$$
e=\widetilde{D}\left(\frac{1-t}{2}\right)
$$

where $D$ is a subgroup of $G$ containing $S$ such that $D / S \subseteq \mathcal{Z}(G / S)$ and $\mathcal{Z}(G / S) /(D / S)$ is cyclic and $t \notin D$.

We now can deduce the structure of the group $\langle u, \varphi(u)\rangle$, where $\varphi$ is an arbitrary involution on $G$.

Theorem 2.4. Let $G \in \mathcal{G}$ and let $u_{x, y}$ and $u_{x^{\prime}, y^{\prime}}$ be non-trivial bicyclic units. Then $\left\langle u_{x, y}, u_{x^{\prime}, y^{\prime}}\right\rangle$ is a free group if and only if there exists a hyperplane $S$ of $G^{\prime}$ such that

1. $t=(x, y) \notin\left(\langle x\rangle \cap G^{\prime}\right) S$,

2. $t^{\prime}=\left(x^{\prime}, y^{\prime}\right) \notin\left(\left\langle x^{\prime}\right\rangle \cap G^{\prime}\right) S$,

3. $t$ is not in $T_{S}$ modulo $S$, where $T_{S}=\left\langle x^{2}, t x^{\prime} x^{-1}\right\rangle$ if $x^{\prime} x^{-1}$ is central modulo $S$, and $T_{S}=\left\langle x^{2}, x^{\prime 2}\right\rangle$ otherwise. 
Otherwise, $\left\langle u_{x, y}, u_{x^{\prime}, y^{\prime}}\right\rangle$ is a torsion-free abelian group.

Proof. Let $S$ be a hyperplane of $G^{\prime}$ satisfying conditions (1) to (3). Condition (1) says that $\langle x S\rangle$ is not normalized by $y S$ and thus the natural image of $u_{x, y}$ is a power of a non-trivial bicyclic unit in $\mathbb{Z}[G / S]$. Similarly, condition (2) says that the natural image of $u_{x^{\prime}, y^{\prime}}$ is a power of a non-trivial bicyclic unit in $\mathbb{Z}[G / S]$. Also $G / S=\langle x S, y S, \mathcal{Z}(G / S)\rangle=\left\langle x^{\prime} S, y^{\prime} S, \mathcal{Z}(G / S)\right\rangle$ and $(G / S) /(\mathcal{Z}(G / S)) \cong C_{2} \times C_{2}$.

It follows that $x^{\prime} S$ equals an element of the from $z_{1} x S, z_{1} y S$ or $z_{1} x y S$ for some $z_{1} \in G$ so that $z_{1} S \in \mathcal{Z}(G / S)$. If, for example $x^{\prime} S=z_{1} x S$ then since $x^{\prime} S$ and $y^{\prime} S$ do not commute, the lack of commutativity in $G / S$ implies that $y^{\prime} S=z_{2} y S$ or $y^{\prime} S=z_{2} x y S$ for some $z_{2} \in G$ so that $z_{2} S \in \mathcal{Z}(G / S)$. The other cases are dealt with similarly. Hence, because of Theorem 2.3 the result follows.

If there does not exist a hyperplane $S$ of $G^{\prime}$ with conditions (1) to (3), then for every hyperplane $S$ of $G^{\prime}$ either $u_{x, y}$ becomes trivial modulo $S$, or $u_{x^{\prime}, y^{\prime}}$ becomes trivial modulo $S$ or the natural images of $u_{x, y}$ and $u_{x^{\prime}, y^{\prime}}$ commute in $\mathbb{Z}[G / S]$. It follows that in every non-commutative simple component $\mathbb{Q}[G] e$ of $\mathbb{Q}[G],\left\langle u_{x, y}, u_{x^{\prime}, y^{\prime}}\right\rangle$ is abelian and hence $\left(u_{x, y}-1\right)\left(u_{x^{\prime}, y^{\prime}}-1\right)$ is nilpotent. It then follows easily from Theorem 2.1 that $\left\langle u_{x, y}, u_{x^{\prime}, y^{\prime}}\right\rangle$ is a torsion-free abelian group.

\section{Examples.}

1. We recover the result of Marciniak and Sehgal for the class of finite groups $G$ which are not Hamiltonian and such that $G / \mathcal{Z}(G) \cong C_{2} \times C_{2}$. Take $x, y \in G$ such that $s=(x, y) \notin\langle x\rangle$, then $u_{x, y}=1+(1-x) y \widehat{x}$ is a non-trivial bicyclic unit. For the classical involution $*, x^{*} x^{-1}=x^{-2}$ is central. Hence $T=\left\langle x^{2}, x^{-2}\right\rangle$, which does not contain $s$ by assumption. Therefore, by Theorem $2.3\left\langle u_{x, y}, u_{x, y}^{*}=u_{s x^{-1}, y^{-1}}\right\rangle$ is free.

2. Consider $u_{b, a}=1+(1-b) a(1+b)$ in $\mathbb{Z}\left[D_{16}^{+}\right]$. Let $\varphi(b)=b$ and $\varphi(a)=a^{5}$, then $T=\{1\}$ and hence $\langle u, \varphi(u)\rangle$ is free. For $\psi(b)=a^{4} b$ and $\psi(a)=a^{3}$, we have that $s \in T=\left\{1, a^{4}\right\}$ and hence $\langle u, \psi(u)\rangle$ is torsion-free abelian.

\section{Subgroups of finite index}

In this section we construct a subgroup of finite index in $\mathcal{U}(\mathbb{Z}[G])$ for $G \in \mathcal{G}$. In order to do so we recall the following definition. 
Definition. [3] For an involution $\varphi$ of $G$, put

$$
\mathcal{U}_{\varphi}(\mathbb{Q}[G])=\{u \in \mathcal{U}(\mathbb{Q}[G]) \mid u \varphi(u)=1\}
$$

and

$$
\mathcal{U}_{\varphi}(\mathbb{Z}[G])=\mathcal{U}_{\varphi}(\mathbb{Q}[G]) \cap \mathbb{Z}[G],
$$

these units are called $\varphi$-unitary. If $\varphi_{1}, \ldots, \varphi_{n}$ all are involutions on $G$, then we put

$$
\mathcal{U}_{\varphi_{1}, \ldots, \varphi_{n}}(\mathbb{Z}[G])=\left\langle\mathcal{U}_{\varphi_{i}}(\mathbb{Z}[G]) \mid i=1, \ldots, n\right\rangle .
$$

We will prove that for each non-commutative Wedderburn component $\mathbb{Q}[G] e_{i}(i=1, \ldots n)$ of $\mathbb{Q}[G]$ there exists an involution $\varphi_{i}$ on $G$ such that the group generated by the Bass cyclic units and $\mathcal{U}_{\varphi_{1}, \ldots, \varphi_{n}}(\mathbb{Z}[G])$ is of finite index in $\mathcal{U}(\mathbb{Z}[G])$. The first part of the proof is done following the same lines of [3], where this result is proved for groups of order 16. For completeness' sake we give a compact version of the argument.

Theorem 3.1. Let $G \in \mathcal{G}$. Denote by $B_{G}$ the group generated by the Bass cyclic units of $\mathbb{Z}[G]$. Then there exist involutions $\varphi_{1}, \ldots, \varphi_{n}$ on $G$ such that

$$
\left\langle B_{G}, \mathcal{U}_{\varphi_{1}, \ldots, \varphi_{n}}(\mathbb{Z}[G])\right\rangle
$$

is a subgroup of finite index in $\mathcal{U}(\mathbb{Z}[G])$.

Proof. First, let $G$ be such that $G / \mathcal{Z}(G) \cong C_{2} \times C_{2}$ and let $x, y \in G$ be such that $G=\langle x, y, \mathcal{Z}(G)\rangle$. Denote by $s$ the unique commutator of $G$. Then by [4, Theorem III.3.3] $G$ has an involution $\varphi$ defined by

$$
\varphi(g)= \begin{cases}g & \text { if } g \text { is central } \\ s g & \text { otherwise }\end{cases}
$$

By $\left[4\right.$, Corollary VI.4.8] $\mathbb{Q}[G] \cong \bigoplus_{i} D_{i}$, a direct sum of fields and generalized quaternion algebras over fields. Let $e_{i}$ be a primitive central idempotent of $\mathbb{Q}[G]$ such that $D_{i}=\mathbb{Q}[G] e_{i}$ and let $O_{i}$ be a $\mathbb{Z}$-order in $D_{i}$. Because $G$ is nilpotent, by [8] the group generated by the Bass cyclic units contains a subgroup of finite index in $\bigoplus_{i} \mathcal{Z}\left(\mathcal{U}\left(O_{i}\right)\right)$. Hence to prove the result it is sufficient to search for a subgroup (of $\varphi$-unitary units) that contains a subgroup of finite index in $S L_{1}\left(O_{i}\right)$, provided $D_{i}$ is a generalized quaternion algebra. Recall that by definition $S L_{1}\left(O_{i}\right)=S L_{1}\left(D_{i}\right) \cap O_{i}$, where $S L_{1}\left(D_{i}\right)$ is the group of elements $q$ of reduced norm $n r(q)=q \bar{q}=1$, where - denotes the 
standard involution with respect to the basis $\left\{e_{i}, x e_{i}, y e_{i}, x y e_{i}\right\}$ of this generalized quaternion algebra. Now for each such $D_{i}$ we have that $\varphi\left(e_{i}\right)=e_{i}$ because the support of $e_{i}$ is central and

$$
\varphi\left(g e_{i}\right)=\overline{g e_{i}},
$$

where $g \in G$. Because - is linear, we get that $\varphi(q)=\bar{q}$ for all $q \in D_{i}$. Hence $S L_{1}\left(D_{i}\right)$ equals the image in $D_{i}$ of the $\varphi$-unitary units of $\mathbb{Q}[G]$. Since general order theory gives us that $\mathcal{U}(\mathbb{Z}[G])$ and $\bigoplus_{i} G L_{1}\left(O_{i}\right)$ have a common subgroup of finite index, we have that $\mathcal{U}(\mathbb{Z}[G])$ contains a subgroup of finite index in each $\left(1-e_{i}\right)+G L_{1}\left(O_{i}\right)$, where $e_{i}$ is the unity of $D_{i}$. Consequently, the $\varphi$-unitary units of $\mathbb{Z}[G]$ contain a subgroup of finite index in each $(1-$ $\left.e_{i}\right)+S L_{1}\left(O_{i}\right)$, as desired.

Now, let $G \in \mathcal{G}$ and let $e_{k}$ be a primitive central idempotent of the rational group algebra $\mathbb{Q}[G]$ determining a non-commutative Wedderburn component. We will show that there exists an involution $\varphi_{k}$ on $G$ that induces the involution (3) on $H=G e_{k}$, in particular $\varphi_{k}\left(e_{k}\right)=e_{k}$. Since the simple components of $\mathbb{Q}[H]$ are simple components of $\mathbb{Q}[G]$ and $H / \mathcal{Z}(H) \cong$ $C_{2} \times C_{2}$, the case above and again order theory, yield the result.

Let $H=\left\langle x_{1}, x_{2}, \mathcal{Z}(H)\right\rangle$, for some $x_{1}, x_{2} \in G$ with $x_{1}^{2}$ and $x_{2}^{2}$ central in $G$. Let $S$ be a hyperplane of the elementary abelian 2-group $G^{\prime}$ that does not contain $t=\left(x_{1}, x_{2}\right)$. Then $e_{k}=\widetilde{D}\left(\frac{1-t}{2}\right)$, where $D$ is a subgroup of $G$ containing $S$ such that $D / S \subseteq \mathcal{Z}(G / S)$ and $\mathcal{Z}(G / S) /(D / S)$ is cyclic and $t \notin D$. As $G / \mathcal{Z}(G)$ is an elementary abelian 2-group, say of rank $n$, we can write $G=\left\langle x_{1}, x_{2}, \ldots, x_{n}, \mathcal{Z}(G)\right\rangle$ with $x_{i}^{2} \in \mathcal{Z}(G), 1 \leq i \leq n$ and $x_{i}$ central modulo $S$ for $3 \leq i \leq n$.

Any element $g \in G$ can be written uniquely as

$$
g=z x_{1}^{a_{1}} x_{2}^{a_{2}} \ldots x_{n}^{a_{n}}
$$

with $z \in \mathcal{Z}(G), a_{i} \in\{0,1\}, 1 \leq i \leq n$. Put $t_{i j}=\left(x_{i}, x_{j}\right)$. Since $G^{\prime}$ is an elementary abelian 2-group we have that $t_{i j}=t_{j i}$. Let $\varphi_{k}: G \rightarrow G$ be given by

$$
\varphi_{k}\left(z x_{1}^{a_{1}} x_{2}^{a_{2}} \ldots x_{n}^{a_{n}}\right)=z t_{12}^{a_{1}+a_{2}} \prod_{i \geq 1}\left(\prod_{j \geq 2, j>i} t_{i j}^{a_{i} a_{j}}\right) x_{i}^{a_{i}}
$$

Notice that the map $\varphi_{k}$ is defined on the generators as $\varphi_{k}\left(x_{1}\right)=t_{12} x_{1}$, $\varphi_{k}\left(x_{2}\right)=t_{12} x_{2}$ and $\varphi_{k}\left(x_{i}\right)=x_{i}$ for all $i \geq 3$. Also $\varphi_{k}(z)=z$ for $z \in \mathcal{Z}(G)$. Note that the support of $e_{k}$ is $D \cup D t$. Suppose that $x_{1}^{a_{1}} x_{2}^{a_{2}} x \in D$ with $x \in\left\langle\mathcal{Z}(G), x_{3}, \ldots, x_{n}\right\rangle$ and $a_{1}, a_{2} \in\{0,1\}$ (but not both equal to zero) then $x_{1}^{a_{1}} x_{2}^{a_{2}} x e_{k}=e_{k}$, thus $x_{1}^{a_{1}} x_{2}^{a_{2}} e_{k} \in \mathcal{Z}(H)$ and therefore $t e_{k}=e_{k}$, a 
contradiction. A similar reasoning holds for $x_{1}^{a_{1}} x_{2}^{a_{2}} x \in D t$. So the support of $e_{k}$ is contained in $\left\langle\mathcal{Z}(G), x_{3}, \ldots, x_{n}\right\rangle$. Hence $\varphi_{k}\left(e_{k}\right)=e_{k}$. Using the fact that $G^{\prime}$ is of exponent 2 we easily can see that $\varphi_{k}$ is an anti-automorphism and that $\varphi_{k}^{2}=1$. Furthermore, if we restrict the involution $\varphi_{k}$ to the simple component $\mathbb{Q}[G] e_{k}$ it induces (3).

\section{References}

[1] S.A. Amitsur, Groups with representations of bounded degree II, Illinois J. Math. 5 (1961), 198-205.

[2] A.A. Borel and Harish Chandra, Arithmetic Subgroups Of Algebraic Groups, Ann. of Math. 75 (1962), 485-535.

[3] A. Dooms, Unitary units in integral group rings, J. Algebra and its Applications, in press.

[4] G. Goodaire, E. Jespers and C. Polcino Milies, Alternative Loop Rings, North Holland, Elsevier Science B. V., Amsterdam, 1996.

[5] B. Hartley and P.F. Pickel, Free Subgroups in the Unit Groups of Integral Group Rings, Canadian J. Math. 32 (1980), 1342-1352.

[6] G. Higman, The Units of Group Rings, Proc. London Math. Soc. (2) 46, (1940), 231-248.

[7] E. Jespers and G. Leal, Generators of Large Subgroups of the Unit Group of Integral Group Rings, Manuscripta Math. 78 (1993), 303315.

[8] E. Jespers, M.M. Parmenter and S.K. Sehgal, Central Units Of Integral Group Rings Of Nilpotent Groups. Proc. Amer. Math. Soc. 124 (1996), no. 4, 1007-1012.

[9] E. Jespers, Á. del Río and M. Ruiz, Groups generated by two bicyclic units in integral group rings. J. Group Theory 5 (2002), no. 4, 493-511.

[10] G. Leal and C. Polcino Milies, Isomorphic groups (and loop) algebras, J. Algebra 155 (1993), 195-210.

[11] Z.S. Marciniak and S.K. Sehgal, Constructing Free Subgroups of Integral Group Ring Units, Proc. AMS 125 (1997), 1005-1009.

[12] A. Pita, Á. del Río and M. Ruiz, Groups of units of integral group rings of Kleinian type, Trans. AMS 357 (2005), no. 8, 3215-3237.

[13] J. Ritter and S.K. Sehgal, Construction Of Units In Integral Group Rings Of Finite Nilpotent Groups, Trans. Amer. Math. Soc. 324 (1991), 603-621. 
[14] A. Salwa, On Free Subgroups Of Units Of Rings, Proc. Amer. Math. Soc. 127 (1999), no. 9, 2569-2572.

[15] S.K. Seghal, Units in Integral Group Rings, Longman Scientific \& Technical Press, Harlow, 1993. 